\title{
De l'angiomatose bacillaire... à la péliose hépatique : des affections liées à de nouveaux agents opportunistes?
}

Trois articles récents, contenus dans la même livraison du New England Journal of Medicine, [1-3] sont consacrés à de nouveaux agents opportunistes qui pourraient être impliqués dans des syndromes cliniques associés ou non au SIDA - tels que : (a) l'angiomatose bacillaire, encore appelée angiomatose épithélioïde [4-6], marquée par des lésions papulo-nodulaires composées d'une prolifération non maligne de petits vaisseaux et de cellules d'aspect épithéloïde ; elle a été initialement décrite dans la peau et les ganglions lymphatiques de sujets séropositifs pour le virus du SIDA, mais peut être étendue, notamment au tube digestif [5] ; lésion pseudo-néoplasique, elle est particulièrement originale par la présence de bacilles, jusque-là non cultivés, colorés par la technique de Warthin-Starry [4, 6], comme dans la maladie des griffes de chat; (b) la péliose hépatique, qui est caractérisée par la présence de cavités kystiques remplies de sang, disséminées dans le parenchyme hépatique ; elle a d'abord été décrite chez des sujets porteurs de tuberculose ou de cancer avancé, puis au cours de la prise prolongée de stéroïdes anabolisants, enfin chez des transplantés rénaux et, très récemment, chez des sujets séro-positifs; (c) ou un syndrome bactériémique prolongé, de cause jusque-là inconnue [4]. Dans deux de ces travaux $[2,3]$, l'agent infectieux n'a pu être cultivé, mais a fait l'objet d'étude par des techniques de génétique moléculaire amplifiant, par PCR, les séquences d'ARN 16S des ribosomes.

Le travail de Slater et al. [1] a conduit à la mise en évidence, par cul$m / s n^{\circ} 3$, vol. 7 , mars 91 ture, d'un agent pathogène jusque-là inconnu, chez cinq sujets (trois immunodéficients dont deux atteints de SIDA, deux sujets immunocompétents) ayant un syndrome fébrile bactériémique prolongé d'étiologie inconnue : après 5 à 15 jours de culture "laborieuse ", l'agent présentait quelques similarités avec des germes connus, notamment - par sa composition en acides gras - avec une rickettsie Rochalimaea quintana (agent de la fièvre des tranchées): d'autres études, notamment celles utilisant des endonucléases de restriction EcoRV ont montré qu'existaient de notables différences phylogénétiques avec ces espèces. Les travaux de Perkocha et al. [2], et de Relman et al. [3] décrivent, quant à eux, la présence d'un micro-organisme - qui est apparemment le même - au cours de l'angiomatose cutanée bacillaire, de la péliose hépatique ou des deux associées, chez 12 malades ayant un déficit immunitaire qui était lié 11 fois à un SIDA : ce germe, vu à l'examen microscopique de biopsies cutanées et/ou hépatiques n'a pu être cultivé. Dans l'un des travaux [3], les séquences d'ARNr $16 \mathrm{~S}$ des ribosomes, étudiés dans les tissus où étaient présents les germes, ont été amplifiées par PCR et semblaient être très voisines sinon identiques chez les quatre malades étudiés; elles étaient similaires à plus de $98 \%$ à celles de $R$. quintana et à plus de $95 \%$ à celles de Brucella abortus. Deux des huit malades atteints de péliose hépatique avaient aussi une angiomatose bacillaire cutanée : les micro-organismes trouvés dans la peau étaient morphologiquement identiques à ceux trouvés dans le foie [2].
Ces résultats sont, comme le souligne Eisenstein dans son éditorial [7], d'un intérêt tout particulier à plusieurs égards: (a) la découverte de micro-organismes ressemblant à $R$. quintana au cours de l'angiomatose bacillaire s'accorde avec la sensibilité à l'érythromycine et aux tétracyclines, récemment décrite, de ce syndrome: les amas de bacilles sont colorés par la technique de WarthinStarry, ressemblant à ceux trouvés dans la maladie des griffes de chat dont l'agent, récemment cultivé, peut aussi être observé au cours du SIDA mais est insensible à l'érythromycine ; (b) l'angiomatose bacillaire peut, dans quelques cas, être cliniquement voire histologiquement confondue avec le sarcome de Kaposi ou avec d'autres néoplasies vasculaires. Sa sensibilité aux antibiotiques justifie sa connaissance par le clinicien; en l'absence de traitement, son évolution peut être fatale ; (c) il est possible que des lésions de péliose hépatique, qui peuvent être observées chez des sujets atteints de déficit immunitaire de type SIDA, soient dues à des bactéries produisant un facteur d'angiogenèse similaire à celui produit dans l'angiomatose bacillaire cutanée; (d) d'un intérêt clinique tout particulier est le rapprochement entre les micro-organismes trouvés dans l'angiomatose bacillaire et la péliose hépatique, et certaines rickettsies ou germes apparentés tels que $R$. quintana (agent de la fièvre des tranchées dont le vecteur est le pou du corps), ou que Bartonella bacilliformis (agent de la fièvre de la Oroya, dont les lésions cutanées - verruga du Pérou - peuvent, au stade chronique, être cliniquement et histologi-

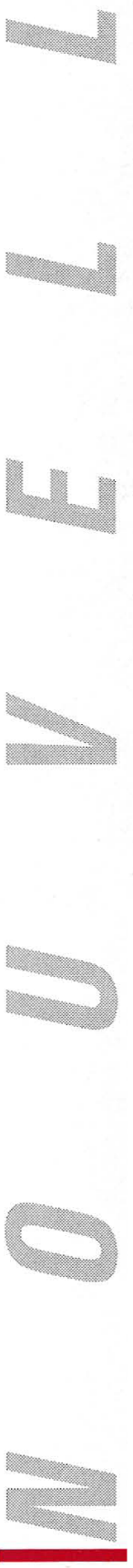


quement identiques à celles de l'angiomatose bacillaire : cet agent a d'ailleurs été proposé comme pouvant être celui de l'angiomatose); si des différences ont été observées avec les séquences d'ARNr $16 \mathrm{~S}$ de $R$. quitana, la comparaison aux séquences d'ARNr $16 \mathrm{~S}$ de $B$. bacilliformis n'a pu être faite car celles-ci ne sont pas encore connues. Il est clair que des travaux ultérieurs utilisant ces techniques de génétique moléculaire en PCR seront d'un très grand intérêt. Finalement, il est ainsi possible que soient mises en évidence chez l'homme - comme cela a été le cas dans des environnements naturels tels que les écosystèmes marins - de nombreuses espèces microbiennes jusque-là non découvcrtes par les méthodes classiques de caractérisation telles que les cultures in vitro.

C. M.

1. Slater IN, Welch DF, Hensel D, Coody DW. A newly recognized fastidious Gram negative pathogen as a cause of fever and bacteremia. N Engl J Med 1990; 323 : 1587-93. 2. Perkocha LA, Geaghan SM, Yen TSB, et al. Clinical and pathological features of bacillary peliosis hepatis in association with human immunodeficiency virus infection. $N$ Engl $J$ Med 1990 ; 323 : 1581-6.

3. Relman DA, Loutit JS, Schmidt TM, Falkow S, Tompkins LS. The agent of bacillary angiomatosis : an approach to the identification of uncultured pathogens. N Engl J Med $1990 ; 323: 1573-80$.

4. Stoler MH, Bonfiglio TA, Steigbigel RT, Pereira M. An atypical subcutaneous infection associated with acquired immune deficiency syndrome. Am J Clin Pathol 1983 ; 80 : 714-8. 5. Cockerell CJ, Whitlow MA, Webster GF, Friedman-Kien AE. Epithelioid angiomatosis : a distinct vascular disorder in patients with the acquired immunodeficiency syndrome or AIDS-related complex. Lancet 1987 ; 2 : 654-6. 6. LeBoit PE, Berger TG, Egbert BM, et al. Epithelioid haemangioma-like vascular proliferation in AIDS : manifestation of cat scratch disease bacillus infection? Lancet 1988; 1 : 960-3.

7. Eisenstein BI. New opportunistic infections, more opportunities. N Engl J Med 1990 ; 323 :

\title{
COURRIER
}

\author{
Tout ne vient pas de Californie
}

L'observation que

la duplication

d'unc partie du

chromosome 21, la

bande 21q22, soit environ 20 millions de paires de bases, peut à ellc seule provoquer le syndrome de la trisomie 21 remonte à 1973 avec les études cytogénétiques de Aula et al. [1]. Les études cliniques avaient montré que le diagnostic de trisomic 21 , confirmée par l'étude caryotypique, nécessitait la présence simultanée de plusicurs signes rassemblés dans un tableau par Jackson et al. [2].

Pris isolément, les signes de ce tableau peuvent être absents chez des individus porteurs d'unc trisomic complètc du chromosome 21 . Sur les 25 signes de ce tableau, lcs individus portcurs de 5 à 12 signes ont unc probabilité de 23 à $84 \%$ d'être trisomique 21 et ceux présentant plus de 13 signes ont une probabilité de $100 \%$ d'être trisomique 21.

Dans notre article [3], nous avons étudié deux individus ayant, l'un, 10 signes et une probabilité de $84 \%$ d'être trisomique 21 et l'autre 13 signes soit une probabilité de $100 \%$ d'être trisomique 21. Ces deux individus sont, selon l'étude cytogénétique, porteurs d'une duplication partielle différente du chromosome 21. Ces patients ont plusieurs signes phénotypiques en commun. L'étude moléculaire nous a permis de définir une zone de moins de 3 millions de pb située en q22.3, dont la duplication serait responsable de ces signes communs. Cet article fut publié en 1989.

Dans l'article commenté [4], dans une brève de $\mathrm{m} / \mathrm{s}\left(\mathrm{n}^{\circ} 10\right.$, vol. 6 , p. 1025) les auteurs étudient une trisomie 21 partielle issuc de la malségrégation d'une translocation $4 ; 21$ parentale aboutissant à une duplication partielle du chromosome 21 (q22.2 et q22.3) et à une délétion

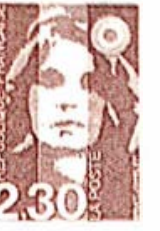

partielle du chromosome 4. De l'étude de deux cas de cette famille, les auteurs en concluent que la région q22.2-q22.3 est la plus petite région connue dont la duplication peut provoquer l'apparition du syndrome de la trisomie 21. Cette région couvre environ 10 millions de $\mathrm{pb}$, soit près de 5 fois la zonc que nous avions précédemment décrite et qui est maintenant appelée DSCR (down syndrome chromosome region) [5]. Plusieurs équipes, dont la nôtre, travaillent maintenant sur la cartographic et le contenu génétique de cette région. Bien entendu, comme nous le mentionnons dans notre publication, le surdosage d'autres gènes, situés hors de la DSCR, doit également contribucr à la pathogénic du syndrome morbide de la trisomic 21.

Jean-Maurice Delabar Pierre M. Sinet

Université René Descartes, faculté de médecine Necker, laboratoire de biochimie génétique, 149, rue de Sères, 75743 Paris Cedex 15, France

1. Aula P, Leisti J, Von Koskull H. Partial trisomy 21. Clin Genet 1973; 4: 241-51. 2. Jackson JF, North ER III, Thomas JG. Clinical diagnosis of Down's syndrome. Clin Genet $1976 ; 9$ : 483-7.

3. Rahmani Z, Blouin JL, Creau-Goldberg $\mathbf{N}$. Critical role of the D21 S55 region on chromosome 21 in the pathogenesis of Down syndrome. Proc Natl Acad Sci USA 1989 ; 86 : 5958-62.

4. Korenberg JR, Kawashima H, Pulst SM. Molecular definition of a region of chromosome 21 that causes features of the Down syndrome phenotype. Am J Hum Genet 1990 ; $47: 236-46$.

5. Carrit B, Litt M. Report of the committee on the genetic constitution of chromosome 20 and 21. Cytogenet Cell Genet 1989; 51 : 358-71. 